\title{
Correlative Light and Electron Microscopic Imaging of Gap Junction Life Cycle Dynamics Using EM Protein Tags and Phospho-Specific Antibodies
}

\author{
Angela Cone ${ }^{1}$, Jeffrey Martell ${ }^{3}$, Thomas Deerinck ${ }^{1}$, Gabriel Cavin ${ }^{1}$, Cinzia Ambrosi ${ }^{1}$, Alice Ting ${ }^{3}$, Mark \\ Ellisman $^{1,2}$ and Gina Sosinsky ${ }^{1,2}$ \\ 1. National Center for Microscopy and Imaging Research, University of California, San Diego CA USA \\ 2. Department of Neurosciences, University of California, San Diego CA USA \\ 3. Department of Chemistry, Massachusetts Institute of Technology, Cambridge, MA, USA
}

Connexin43 (Cx43) is a ubiquitous and critical gap junction protein, playing important regulatory and developmental roles in many tissues including heart, brain and various endothelium, among others. It has a short half-life of $~ 3-6$ hours and is constantly turning over concomitant with gap junction plaque remodeling. Its C-terminus contains at least 12-13 serines that get phosphorylated at key steps in its life cycle by different kinases that are parts of larger signaling cascades [1]. These specifically phosphorylated serines act as tags, gatekeepers or signals to other proteins. During the cell cycle, kinasemediated gap junction intercellular communication, morphology and distribution of Cx43-containing structures change dramatically. We previously showed that during mitosis, $\mathrm{Cx} 43$ is internalized and recycled in a highly coordinated process with different populations of phospho-isoforms localized to specific cellular compartments [2]. Even within gap junction plaques (seen as punctate staining around adjoining cells), phosho-form segregation occurs as shown in Figs. 1A and 1B where pSer368-Cx43 forms sub-domains after Protein Kinase C (PKC) activation by Phorbol 12,13-dibutyrate (PdBu) of endogenously expressing NRK cells and detected by a pSer368 anti-peptide phospho-specific antibody.

While gap junctions have a distinct morphology in electron micrographs, identification of subpopulations of $\mathrm{Cx} 43$ bearing cytoplasmic vesicles, ER, Golgi, lysosomes or gap junctions during the cell cycle requires protein tags to highlight them from the overall population of vesicles with the highest resolution possible. The combined use of correlated light and electron microscopic (CLEM) genetically appended tags and $\mathrm{Cx} 43$ serine phosphorylation specific antibodies with an emphasis on 3D visualization adds another level of mechanistic understanding to our knowledge gap in Cx43 regulation. Genetically appended tags that highlight specific proteins of interest help draw attention to trafficking intermediates in their complex cellular environment. Recently, two protein EM tags have been developed independently, appended to Cx43 and imaged with CLEM. MiniSOG is an efficient single oxygen generator driven by light to polymerize DAB [3]. Cx43-miniSOG is first photooxidized in the presence of $\mathrm{DAB}$ and then prepared for and imaged with EM. $A P E X$, is a mutagenized version of ascorbate peroxidase (APX), a class I cytosolic plant peroxidase [4]. APX catalyzes the $\mathrm{H}_{2} \mathrm{O}_{2}$-dependent polymerization of 3,3'- diaminobenzidine (DAB) into a localized precipitate that gives EM contrast after treatment with $\mathrm{OsO}_{4}$, similar to Horse Radish Peroxidase staining. APEX is monomeric, has increased catalytic activity from APX and is active after fixation. Imaging tags typically appended to the Cterminus of Cx43 have similar trafficking kinetics and localizations as well as full channel activity. When combined with a fluorescent protein such as GFP (Fig. 1C), APEX has the capability for CLEM imaging (Fig. 1D) and can be further combined with phospho-specific antibodies using immunomethods for LM or EM detection. APEX has the advantage of obtaining staining over larger areas of cells or tissues that makes it especially attractive for our goal where we want obtain galleries of high resolution large scale EM volumes [5,6], particularly for analyzing with large numbers of specifically stained $\mathrm{Cx} 43$ in different stages of mitotic cells in order to quantitate their distributions. 
References:

[1] J. L. Solan, P. D. Lampe. Biochem J 419, 261 (2009).

[2] D. Boassa et al. Traffic 11, 1471 (2010).

[3] X. Shu et al. PLoS Biol 9, e1001041 (2011).

[4] J. D. Martell et al. Nat Biotechnol 30, 1143 (2012).

[5] S. Phan et al. J. Struct. Biol. 180, 154 (2012).

[6] J. B. West et al. Respir Physiol Neurobiol 170, 202 (2010).

[7] Funding was provided by NIH grants DP1 OD003961 (A.Y.T.), P41GM103412 (M.H.E.),

GM065937 (G.E.S.), GM072881 (G.E.S.). J.D.M. was supported by NSFGR and NDSEG fellowships.
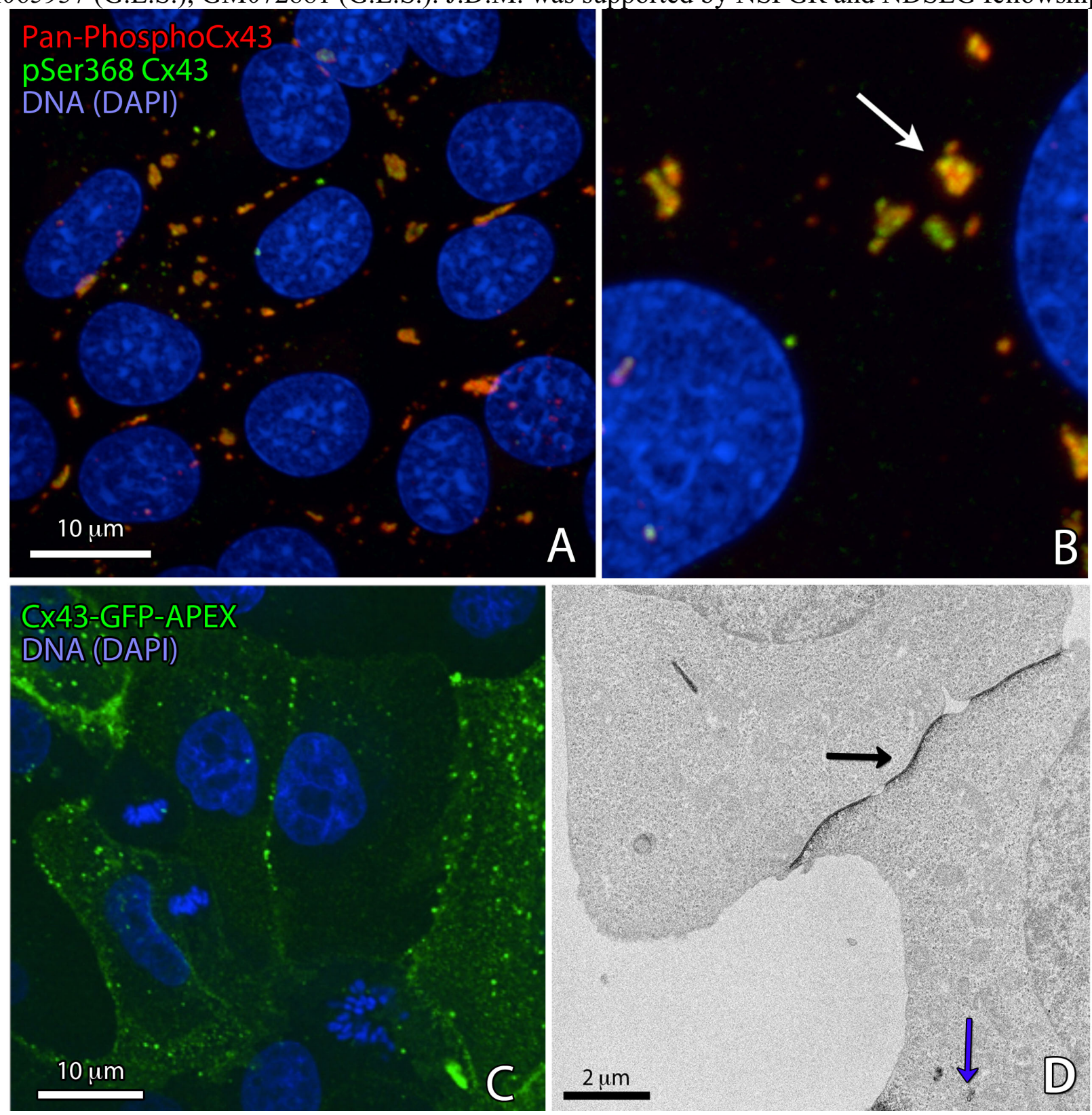

Figure 1. (A) Immunolabeling of Cx43 in NRK cells with a pan-phospho antibody and an antibody that stains only pSer368-Cx43. (B) Higher magnification view showing segregation. (C) GFP fluorescence from MDCK cells tagged with Cx43-GFP-APEX. (D) EM image of Cx43-GFP-APEX in HEK293 cells showing specific darker staining. Black arrow = Large gap junction. Blue arrow = intracellular vesicles. 